\title{
Stimulants augmentation with an antidepressant for the treatment of the major depressive disorder (MDD)
}

\author{
*Dr. Eva R. Haddad, Dr. Muath F. Marashdeh, Dr. Maxim A. Obaisat, Dr. Malik M. Al-Alwan \\ ${ }^{1}$ Pharmacist, RMS, \\ ${ }^{2,3,4} \mathrm{MD}$, Psychiatry, Jordanian Royal Medical Services,
}

\begin{abstract}
:
The use of antidepressants and sedative drugs is the best treatment proved for major depressive disorder and in some cases the electrical convulsion therapy. The use of stimulants off-label in clinical practice an increasing concern as there is no research evidence to support their effectiveness.

Objectives:

The aim of the study to describes the role of stimulant in the treatment of major depressive disorder specifically its risks, benefits and potential use as well as antidepressants. Clinically, the rapid improvement of depressive symptoms with stimulants is often dramatic but short-term, indicating that they are likely to work through different mechanisms of traditional antidepressants. More importantly, there is little evidence from randomized controlled trials that support their effectiveness in the treatment of depression, although stimulants have been shown to reduce the symptoms of prominent depression, such as fatigue. Research is urgently needed to clarify the mechanisms of stimulants and evaluate their longterm benefits and risks in treating major depression. Ultimately, the specificity of the procedure should be determined to inform the evolving clinical use of stimulants compensation with antidepressants in the management of major depression. Until then, they should be described only if they are absolutely necessary, even if their prescriptions must be simple and time-bound unless they are for investigation purposes.
\end{abstract}

\section{Method:}

Randomized controlled trials (RCTs) of one year follow-up 120 adult patients diagnosed with major depressive disorder (MDD) using the Hamilton-Depression Scale (HAM-D) from Jun 2017 to January 2018 of stimulants as augmenting agents with antidepressants (SSRIs) for MDD. A descriptive analysis by source, sex, age, and type of report was performed.

Results:

Of one hundred twenty patient's participant in our study:

Sixty five (54.2\%) patients shows highly improvement in daily life activity and depressive symptoms within first week of augmentation agent and became in remission with continues on therapeutic dose of medication.

Forty nine (41.6\%) patients shows highly improvement in daily life activity and depressive symptoms within second to third week of augmentation agent and psychotherapy then became in remission with continues on therapeutic dose of medication and psychotherapy.

two $(1.4 \%)$ patients not responding to medication with 4 weeks of use than the diagnosis reviewed, proper physical examination was done by medical doctor after that the diagnoses of MDD proved after that they referred for Electro convulsive therapy (ECT) shows improvement post third session in daily life activity and depressive symptoms and after $9^{\text {th }}$ session became in remission with continues on therapeutic dose of medication. Four $(2.8 \%)$ patients left the study without giving reasons as there rights in consent form.

\section{Conclusion:}

We conclude that the augmentation agent is necessary to quick improve daily activity living of MDD patients based on clinical practice and trial study's as well as augmentation agent individually FDA proved and no risk of drug-drug interaction during augmentation.

The psychiatrist and other medical personnel must share the effectiveness of clinical trials and awareness 
with the FDA about the success of the clinical augmentation agent.

Key words: Major Depression, Antidepressants, Methylphenidate, Augmentation, effectiveness of treatment.

\section{Introduction:}

Depression is a mood disorder that causes a persistent feeling of sadness and loss of interest. Also called major depressive disorder or clinical depression, it affects how person feel, think and behave and can lead to a variety of emotional and physical problems. Patient may have trouble doing normal day-to-day activities, and sometimes he may feel as if life isn't worth living.

Depression may occur only once during humane life, people typically have multiple episodes. During these episodes, symptoms occur most of the day, nearly every day and may include: ${ }^{1,2}$

- Feelings of sadness, tearfulness, emptiness or hopelessness

- Angry outbursts, irritability or frustration, even over small matters

- Loss of interest or pleasure in most or all normal activities, such as sex, hobbies or sports

- Sleep disturbances, including insomnia or sleeping too much

- Tiredness and lack of energy, so even small tasks take extra effort

- Reduced appetite and weight loss or increased cravings for food and weight gain

- Anxiety, agitation or restlessness

- Slowed thinking, speaking or body movements

- Feelings of worthlessness or guilt, fixating on past failures or self-blame

- Trouble thinking, concentrating, making decisions and remembering things

- Frequent or recurrent thoughts of death, suicidal thoughts, suicide attempts or suicide

- Unexplained physical problems, such as back pain or headaches

More than just a bout of the blues, depression isn't a weakness and you can't simply "snap out" of it. Depression may require long-term treatment. But don't get discouraged. Most people with depression feel better with medication, psychotherapy or both.

Treatment major depression is, unfortunately, a common variant of major depressive disorder (MDD) and a significant source of referrals from primary care physicians to psychiatrists. Despite initial adequate antidepressant mono-therapy of appropriate trial duration and dose optimization many patients will not fully respond or remit into a wellness state, possibly "due to varying degrees of resistance to treatment. ${ }^{3,4}$ In fact, the longer a depressive episode lasts, as complicating factors increase, more treatments are failed, then resistance tends to increase and prognosis decreases. ${ }^{5,6}$ Similar to other mental illnesses, such as psychotic processes and bipolar disorder, a failure to obtain remission of depressive symptoms may neurophysiologically predispose an individual to an increase in relapses with a worsening of symptoms in future In order to combat MDD, many psycho-pharmacologists choose to switch antidepressant medications in order to continue a mono-therapy approach, while others opt to add or combine agents together. For discussion purposes, an augmentation agent is used when said agent is not FDA approved for the treatment of MDD, but is used in an off-label manner to gain remission. As the term augmentation suggests, an agent is prescribed and utilized simultaneously and in conjunction with an already therapeutically dosed, FDAapproved antidepressant. A combination strategy utilizes two FDA-approved antidepressants added together in order to gain better remission of symptoms. In general, augmentation and combination strategies do not usually involve the simultaneous start of two agents, but rather assumes that the first agent used has not yielded a remission of symptoms and, therefore, a second agent is added in an augmentation or combination fashion secondarily.

There is no definitive proof that either strategy is safer or more effective, but it appears in clinical practice that primary care clinicians are more likely to switch agents, while mental health clinicians are more likely to add or combine agents. Irrespective of the chosen treatment strategy, the goal in treatment of the MDD patient should be the remission of depressive symptoms and, ideally, recovery. Remission is defined as the elimination of all depressive symptoms, as defined by an inventory or scale, such as the Hamilton Depression Rating Scale (HDRS) or the Beck Depression Inventory (BDI), while recovery represents a state of "wellness" sustained for several months. Remission should be considered the goal of optimal treatment of depressive symptoms, as response, or a 50-percent reduction in symptoms similarly assessed, neither equates to patient's subjective sense of well health, nor does it optimally minimize a patient's risk for future depressive episodes. ${ }^{6}$ 
Given the tendency to combine agents without bona fide FDA approvals, in an effort to obtain symptom remission rather than settling for a patient treatment response, a clinician must examine the data available to support this practice. Unfortunately, these data rarely include double-blind, randomized, placebo-controlled trials that represent the gold standard and most stringent of study designs with the greatest minimization of bias in deriving results of high validity. It is often important for clinicians to participate in continuing medical education conferences and workgroups to remain abreast with the constantly evolving community standard of care regarding these practices, as rigorous studies are not often available in regards to these complex prescribing strategies.

We have written this article in order to describe the existing evidence base in regards to augmentation and combination strategies employed in the treatment of MDD. In addition, this review attempts to apply an evidence-based formulation with which to consider and evaluate MDD prescribing practices.

\section{Method:}

Randomized controlled trials (RCTs) of one year follow-up conducting 120 adult patients diagnosed with major depressive disorder (MDD) using the Hamilton-Depression Scale (HAM-D) 62 of which males $(51.7 \%)$ and 58 females $(48.3 \%)$. All participants were above age of 17 years, the eldest was 57 years old during the period of Jun 2017 to January 2018.

Stimulant medications prescribed as augmenting agents with antidepressants (SSRIs) for MDD. A descriptive analysis by source, sex, age, and type of report was performed at Al-Rashid mental health care hospital which is the largest privet referral hospital in Jordan. Investigations included MDD-Score ${ }^{\mathrm{TM}}$ Blood test and other relevant tests where necessary to rule-out other medical condition causing MDD. Participant's selection was based on the formerly mentioned points, and we enrolled the one-hundred twenty patients in the hospital clinic. All patients agreed to participate, after suitable explanations about the nature and aim of the study. Non-structured but comprehensive psychiatric interviews were conducted for each patient, relying on (DSM-V) as a diagnostic tool ${ }^{1}$. Then the standardized Arabic version of Hospital Depression Scale was applied to all patients.

\section{Results:}

\section{Augmentation Strategies}

As introduced earlier, an augmentation agent is used when said agent is not FDA approved for the treatment of MDD, but, rather, it is used in an off-label manner to enhance the effect of an initial antidepressant. The augmentation agent is added to a current therapeutically dosed FDA approved antidepressant to bolster effectiveness. Typically, the goal of augmentation strategies is to obtain symptom remission more rapidly when such remission cannot be obtained by optimizing mono-therapy.

Psycho-stimulants, such as methylphenidate, are typically utilized in the treatment of attention disorders, most notably in the pediatric and adolescent patient population. The efficacy of stimulants is based in their actions as either inhibitor of dopamine/norepinephrine reuptake, or release of these monoamines into the synaptic cleft. ${ }^{7}$ This dopaminergic boost acting on the level of mesolimbic dopaminergic projections is believed to contribute to an enhanced and rapid antidepressant effect and possibly addictive potential. ${ }^{7}$

Open-label studies by Lavretsky and colleagues ${ }^{8,9}$ and Gwirtsman ${ }^{10}$ showed methylphenidate (MPH) 5 to $20 \mathrm{mg} /$ day to be an effective augment of citalopram and TCAs, respectively, with 20- to 60-percent treatment response by the end of Week 2. In both the Gwirtsman study and Fawcett and colleagues' retrospective pemoline or dextroampthetamine augmentation of MAOi study, 70 to $80 \%$ of the study populations that were not limited to the elderly as in Lavretsky trials showed "good responses" and improvement in CGI scores by the end of the trials. ${ }^{10,11}$

While side effects were generally mild in these studies, usually limited to sedation, nausea, and anxiety, more significant adverse events were reported. Three patients developed manic episodes, three patients required the cessation of the stimulant, and several patients required dosage adjustments to maintain tolerability. ${ }^{8-11}$

Comments: While there remains to be any positive data from randomized double-blind trials, multiple openlabel trials have suggested that methylphenidate at doses between 5 to $20 \mathrm{mg} /$ day and other stimulants are efficacious antidepressant augments and treatment accelerators, notably in depressed elderly patients. Unfortunately, well-documented adverse effects, such as anxiety, insomnia, dose-dependent hypertension, abuse potential, and exacerbation of psychosis and mania, could continue to limit their use in the absence of more robust data regarding both long-term efficacy and tolerability.

In our study of one hundred twenty participants diagnosed as MDD: 
$65(54.2 \%)$ patients shows highly improvement in daily life activity and depressive symptoms within first week of augmentation agent and became in remission with continues on therapeutic dose of medication.

49 (41.6\%) patients shows highly improvement in daily life activity and depressive symptoms within second to third week of augmentation agent and psychotherapy then became in remission with continues on therapeutic dose of medication and psychotherapy.

$2(1.4 \%)$ patients not responding to medication with 4 weeks of use than the diagnosis reviewed, proper physical examination was done by medical doctor after that the diagnoses of MDD proved after that they referred for Electro convulsive therapy (ECT) shows improvement post third session in daily life activity and depressive symptoms and after $9^{\text {th }}$ session became in remission with continues on therapeutic dose of medication.

$4(2.8 \%)$ patients left the study without giving reasons as there rights in consent form.

\section{Discussion:}

All referred cases with MDD in the psychiatry clinic were considered to have no organic cause which has already been excluded by start of our study. We suggest given the imbalance between usual polypharmacy practices and the amount of available controlled data that some standards of accepted rational poly-pharmacy be developed. It may be possible to use pharmacodynamic theory (how each drug works based on neurotransmitter and receptor profile) in order to predict which agents may be most likely to be safe and effective when used together. Obviously many of the agents individually have safety and tolerability data given that they have gone through an FDA-approval process, which suggests reasonable efficacy, tolerability, and safety data from multiple clinical research trials. When there is little controlled data and no clear pharmacodynamic rationale available to support combining or augmenting drugs, then at least the available mono-therapy efficacy and safety data must be consulted and weighed before prescribing occurs.

A clinician must weigh the availability of FDA approvals, strength of evidence-based combination/augmentation data, and pharmacodynamic theory before prescribing. When there is a lack of evidence in any of these areas, clinicians should be aware of the usual clinical practices in their community or even nationally. This is usually accomplished by participating in local and national continuing medical education (CME) events, attending area grand rounds, or APA branch meetings.

Concurrently though the idea that "the most common augmentation strategies in depression are those with the least controlled evidence" highlights the fact that much of psychopharmacology is also an "art" in which clinicians prescribe based on anecdotal experience of positive patient responses based on particular depressive symptoms. Similar to the idea of nature and nurture, where both are necessary to adequately explain human processes, a consideration of both the clinician's subjective experience and more objective evidence-based data is necessary. Weighing all of these issues and with the goal of gaining depressive remission in our patients, clinicians may then make well-rounded decisions when prescribing for their patients with major depressive disorder.

\section{Conclusion:}

We conclude that the augmentation agent is necessary to quick improve daily activity living of MDD patients based on clinical practice and trial study's as well as augmentation agent individually FDA proved and no risk of drug-drug interaction during augmentation.

The psychiatrist and other medical personnel must share the effectiveness of clinical trials and awareness with the FDA about the success of the clinical augmentation agent.

\section{References:}

[1] Diagnostic and Statistical Manual of Mental Disorders, Fifth Edition, American Psychiatric Association, Arlington, VA. (2013).

[2] Kaplan and Sadock's Synopsis of Psychiatry: Behavioral Sciences/Clinical Psychiatry Eleventh Edition. By Benjamin J. Sadock (Author), Virginia A. Sadock (Author), Dr. Pedro Ruiz MD (Author)

[3] Fava M, Davidson KG. Definition and epidemiology of treatment-resistant depression. Psychiatr Clin of N Am. 1996;19(2):179-200. [PubMed] [Google Scholar] 
[4] O'Reardon J, Brunswick D, Amsterdam J. Treatment-resistant depression in the age of serotononin: Evolving strategies. Curr Opin Psychiatry. 2000;13:93-8. [Google Scholar]

[5] Hirschfeld RM, Keller MB, Panico S, et al. The National Depressive and Manic-Depressive Association consensus statement on the undertreatment of depression. Jama. 1997;277:333-40. [PubMed] [Google Scholar]

[6] Thase ME. Defining remission in patients treated with antidepressants. J Clin Psychiatry. 1999;60(suppl 22):3-6. [PubMed] [Google Scholar]

[7] Stahl S. Essential Psychopharmacology: Neuroscientific Basis and Practical Applications. Second Edition. Cambridge: Cambridge University Press; 2000. [Google Scholar]

[8] Lavretsky H, Kim MD, Kumar RA. Combined treatment with methylphenidate and citalopram for accelerated response in the elderly: An open trial. J Clin Psychiatry. 2003;64(12):1410-14. [PubMed] [Google Scholar]

[9] Lavretksy H, Kumar A. Methylphenidate augmentation of citalopram in elderly depressed patients. Am J Geriatr Psychiatry. 2001;9:298-303. [PubMed] [Google Scholar]

[10] Gwirtsman H, Szuba MP, Toren L, Feist M. The antidepressant response to tricyclics in major depressives is accelerated with adjunctive use of methylphenidate. Psychopharmacol Bull. 1994;30(2):157-64. [PubMed] [Google Scholar]

[11] Fawcett J, Kravitz HM, Zajecka JM, Schaff MR. CNS stimulant potentiation of monoamine oxidase inhibitors in treatment refractory depression. J Clin Psychopharmacol. 1991;11(2):127-32. [PubMed] [Google Scholar] 